\title{
Fecal elastase levels in children diagnosed with functional abdominal pain-not otherwise specified
}

\author{
Burcu Güven ${ }^{1 \oplus}$, Nuriye Burçin Turan ${ }^{2 \oplus}$, Ahmet Fayik Öner ${ }^{3 \oplus}$, Kamuran Karaman ${ }^{3 \oplus}$, \\ Mecnun Çetin ${ }^{4 \oplus}$ \\ ${ }^{1}$ Department of Pediatric Gastroenterology, Karadeniz Technical University Faculty of Medicine, Trabzon; Divisions ${ }^{3}$ Pediatric \\ Hematology and ${ }^{4}$ Pediatric Cardiology, ${ }^{2}$ Department of Pediatrics, Yüzüncü Yıl University Faculty of Medicine, Van, Turkey.
}

\begin{abstract}
Background. Although the exact pathophysiology of functional gastrointestinal diseases remains unclear, numerous etiologies have been blamed, including visceral hypersensitivity, gastrointestinal motility disorders, psychological factors, intestinal mucosal inflammation, intestinal microbiota, and post-infectious syndromes. In the present study, we aimed to evaluate pancreatic insufficient patients diagnosed with functional abdominal pain-not otherwise specified (FAP-NOS) according to Rome IV criteria.
\end{abstract}

Methods. The study included a total of 110 patients aged 4-17 years who were diagnosed with FAP-NOS according to Rome IV criteria. The control group consisted of 80 patients with no gastrointestinal disorders and chronic diseases. Glucose, amylase, lipase, pancreatic amylase, immunoreactive trypsinogen (IRT) and fecal elastase (FE-1) levels were examined for each patient.

Results. No significant difference was found between the two groups with regard to lipase, pancreatic amylase, IRT, and serum glucose levels. However, the amylase levels were significantly higher and the FE-1 levels were significantly lower in the study group compared to the control group $(p=0.007$ and $p<0.001)$. The cut-off value detected in in ROC analysis for the diagnostic value of FE-1 in predicting FAP-NOS was found to be 140.107 $\mu \mathrm{g} / \mathrm{g}$. Based on this value, the sensitivity, specificity, PPV, and NPV of FE- 1 were $82.1 \%, 66.2 \%, 77 \%, 73 \%$, respectively. Accordingly, the likelihood of FE-1 in providing a positive value in patients with FAP-NOS was almost 9 times higher than in individuals without FAP-NOS.

Conclusions. FE-1 levels were significantly lower in children diagnosed with FAP-NOS and we consider that this difference could be attributed to malabsorption secondary to dysbiosis as there is not enough data.

Key words: FAP-NOS, fecal elastase, pancreas insufficiency.

Almost $35-38 \%$ of elementary school children are admitted to hospital once a week due to abdominal pain. ${ }^{1,2}$ Of these admissions, only one-third are diagnosed as functional abdominal pain disorders (FAPD). ${ }^{3}$ Although the exact pathophysiology of functional gastrointestinal diseases (FGIDs) remains unclear, numerous etiologies have been blamed, including visceral hypersensitivity, gastrointestinal motility disorders, psychological factors, intestinal

$\bowtie$ Burcu Güven

burcuguven55@gmail.com

Received 25th March 2020, revised 3rd June 2020, accepted 7th June 2020. mucosal inflammation, intestinal microbiota, and post-infectious syndromes. ${ }^{4}$

Chronic pancreatitis (CP) is considered an extremely rare condition in the general population. However, postmortem studies have indicated that the disease is not as rare as expected and that it has a prevalence of $6-12 \%$ in the general population and many patients remain undiagnosed. ${ }^{5}$ Patients with $\mathrm{CP}$ and those with FAP, particularly those with diarrhea-predominant irritable bowel syndrome (IBS-D), often present to hospital with similar complaints such as abdominal pain and diarrhea. In such patients, however, routine pancreatic function tests and pancreatic 
imaging techniques are not recommended as per 2016 Rome IV criteria. ${ }^{6}$

Leeds et al. ${ }^{7}$ evaluated patients with IBS-D and found that $19(6.1 \%)$ of them had low fecal elastase-1 (FE-1) levels. Talley et al. ${ }^{8}$ reported that one in 50 patients that had otherwise unexplained abdominal pain and/or diarrhea or IBS-D were diagnosed with pancreatic exocrine insufficiency (PEI). ${ }^{8}$ In both of these studies, most of the patients were at advanced ages and alcohol abuse was blamed as the most common etiology. Nevertheless, to our knowledge, there have been no such studies conducted on children.

According to Rome IV criteria, abdominal pain that does not otherwise fit a specific disease such as irritable bowel syndrome, functional dyspepsia, and abdominal migraine is termed as functional abdominal pain-not otherwise specified (FAP-NOS). ${ }^{3}$ In the present study, we aimed to evaluate pancreatic function test results in patients diagnosed with FAP-NOS according to Rome IV criteria.

\section{Material and Methods}

The study included a total of 110 patients aged 4-17 years who were admitted to our pediatric outpatient clinic and were diagnosed with FAPNOS according to Rome IV criteria between April 1 and September 1, 2019. The control group consisted of 80 patients with no gastrointestinal disorders and chronic diseases. However, two patients in the study group and three patients in the control group were excluded from the study due to technical difficulties. As a result, a total of 185 patients comprising 108 patients with FAP-NOS and 77 control subjects were included in the study.

Age, gender, and body weight and height were recorded for each patient. The standard deviation score (SDS) for body weight was also calculated. ${ }^{9}$

Serum samples were obtained from each patient and were analyzed for glucose, amylase, lipase, and pancreatic amylase levels. The measurements were performed spectrophotometrically, using a Beckman Coulter AU5800 autoanalyzer. Additionally, 3 cc of blood samples were taken for the measurement of immunoreactive trypsinogen (IRT) levels and were collected into biochemical tubes containing no anticoagulants. Within the first hour after the collection of blood samples, the tubes were centrifuged at 3,000 rpm for 20 min $+4{ }^{\circ} \mathrm{C}$ and the supernatants were placed in Eppendorf tubes and then were stored at -80 ${ }^{\circ} \mathrm{C}$ until analysis. The samples were analyzed concurrently by Ylbiont ELISA Kit (range, 2-600 ng/L; sensitivity, $1.01 \mathrm{ng} / \mathrm{L}$ ).

A single stool sample was obtained from each patient and was stored at $-20{ }^{\circ} \mathrm{C}$. Prior to the analysis, the samples were transferred to the laboratory and were thawed at $2-8{ }^{\circ} \mathrm{C}$ and then kept at room temperature for one hour. After achieving room temperature, the samples were analyzed concurrently by Ylbiont ELISA Kit (range, 20-6000 ng/L; sensitivity, $10.15 \mathrm{ng} / \mathrm{L}$ ).

\section{Statistical analysis}

Data were analyzed using IBM SPSS for Windows version 22.0 (IBM Corp. Released 2013. IBM SPSS Statistics for Windows, Version 22.0. Armonk, NY: IBM Corp.). Descriptive statistics were expressed as mean \pm standard deviation (SD) for parametric data, as median (minimum-maximum) for nonparametric data, and as frequencies (n) and percentages (\%) for categorical data. Variables were compared using Student's t-test for parametric data (t-test for independent variables), Mann-Whitney $U$ test for nonparametric data, and Pearson's chisquared test for categorical data. The diagnostic performance of FE-1 in predicting FAP-NOS was analyzed using Receiver Operating Characteristic (ROC) analysis and its accuracy was analyzed using the area under curve (AUC) obtained from ROC analysis. The optimal cutoff value of FE-1 was calculated according to the Youden Index. Based on the optimal cut-off value, sensitivity, specificity, negative predictive value (NPV), positive predictive 
value (PPV), positive prediction rate, negative prediction rate, likelihood ratio (LR), positive likelihood ratio $(\mathrm{LR}+)$, negative likelihood ratio $(\mathrm{LR}+)$, and odds ratio were calculated. A $p$ value of $<0.05$ was considered significant.

The study was approved by Institutional Ethics Committee of Yüzüncü Yıl University (Approval Date: March 19, 2019; No. 2019/07). A written informed consent was obtained from each parent/guardian.

\section{Results}

The study included a total of 185 patients, comprising 95 (51.4\%) boys and 90 (48.6\%) girls with a mean age of $8.92 \pm 3.41$ (median, 8) years. No significant difference was found between the study and control groups with regard to age and gender ( $p=0.524$ and $p=0.302$, respectively) (Table I). Similarly, no significant difference was found between the two groups with regard to lipase, pancreatic amylase, IRT, and serum glucose levels $(p=0.672, p=0.432, p=0.110, p=$ 0.801 , respectively) (Table I). However, the amylase levels were significantly higher and the FE-1 levels were significantly lower in the study group compared to the control group ( $p=0.007$ and $p<0.001$, respectively) (Table I, Figs 1-2).

The cut-off value detected in in ROC analysis for the diagnostic value of FE-1 in predicting FAP-
NOS was found to be $140.107 \mu \mathrm{g} / \mathrm{g}$ (Fig. 3). Based on this value, the sensitivity, specificity, PPV, and NPV of FE-1 were $82.1 \%, 66.2 \%, 77 \%$, and $73 \%$, respectively. Moreover, the false positive rate was 0.179 and the LR+ and LR- values were 2.43 and 0.278 , respectively. Accordingly, the likelihood of FE-1 in providing a positive value in patients with FAP-NOS was almost 9 times higher than in individuals without FAP-NOS. On the other hand, a significant odds ratio was obtained for the diagnostic value of FE-1 in predicting FAP-NOS (95\% Confidence Interval [CI]: 4.52-17.82).

Nevertheless, no significant correlation was found between FE-1 and amylase, lipase, pancreatic amylase, and IRT levels in the study group $(\mathrm{r}=0.49, \mathrm{r}=0.147, \mathrm{r}=0.044, \mathrm{r}=0.036$, respectively) ( $p>0.05$ for all).

\section{Discussion}

Clinical manifestations of PEI may vary according to underlying etiologies, stage of disease, nutritional status, and numerous other factors. ${ }^{10,11}$ However, patients with PEI often present with typical symptoms such as steatorrhea and weight loss, most of which can be observed in the late stage of the disease. ${ }^{12}$ In early stages, however, patients may present with nonspecific symptoms such as abdominal distension and pain. ${ }^{13}$ In line with this data,

Table I. Demographic and biochemical characteristics.

\begin{tabular}{lccc}
\hline & $\begin{array}{c}\text { Study group } \\
(\mathrm{n}=108)\end{array}$ & $\begin{array}{c}\text { Control group } \\
(\mathrm{n}=77)\end{array}$ & $p$ \\
\hline Age (years) & $8(4-17)$ & $9(4-17)$ & $0.347^{1}$ \\
Gender (female. \%) & $56(51.9 \%)$ & $34(44.2 \%)$ & $0.302^{2}$ \\
Body weight SDS & $0.205(-0.6-1.8)$ & $0.36(-0.4-2.1)$ & $0.064^{1}$ \\
Lipase (U/L) & $17(5-77)$ & $16(7-36)$ & $0.672^{1}$ \\
Amylase (U/L) & $75(34-144)$ & $64(14-109)$ & $0.007^{1}$ \\
Pancreatic amylase (U/L) & $20(3-50)$ & $20(10-41)$ & $0.432^{1}$ \\
IRT $(\mu \mathrm{g} / \mathrm{L})$ & $73.42(20.2-338)$ & $56.4(12.05-338.52)$ & $0.110^{1}$ \\
Fecal elastase $(\mu \mathrm{g} / \mathrm{g})$ & $122.1(22.49-216.59)$ & $147.85(24.75-240.25)$ & $<0.001^{1}$ \\
Glucose $(\mathrm{mg} / \mathrm{dl})$ & $88.2 \pm 8.447$ & $87.9 \pm 9.725$ & $0.801^{3}$ \\
\hline
\end{tabular}

${ }^{1}$ : Mann-Whitney U test, median (minimum-maximum), ${ }^{2}$ : Pearson's Chi-squared test ${ }^{3}$ : Student's t-test IRT: immunoreactive trypsinogen 


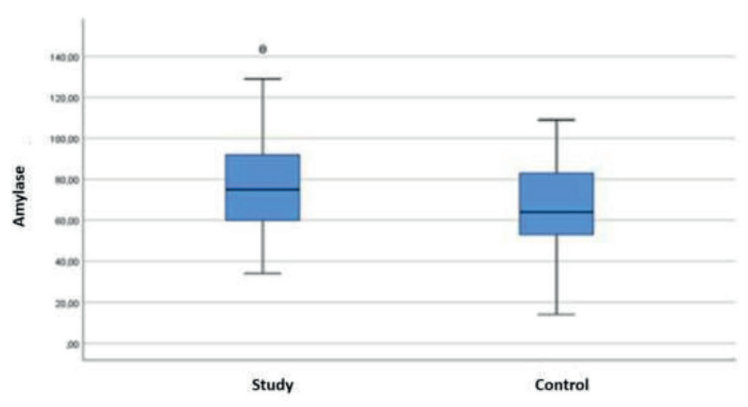

Fig. 1. Amylase levels in both groups.

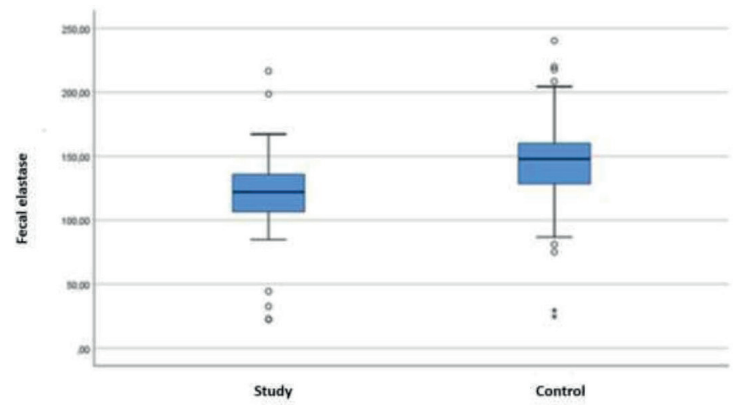

Fig. 2. Fecal elastase levels in both groups.

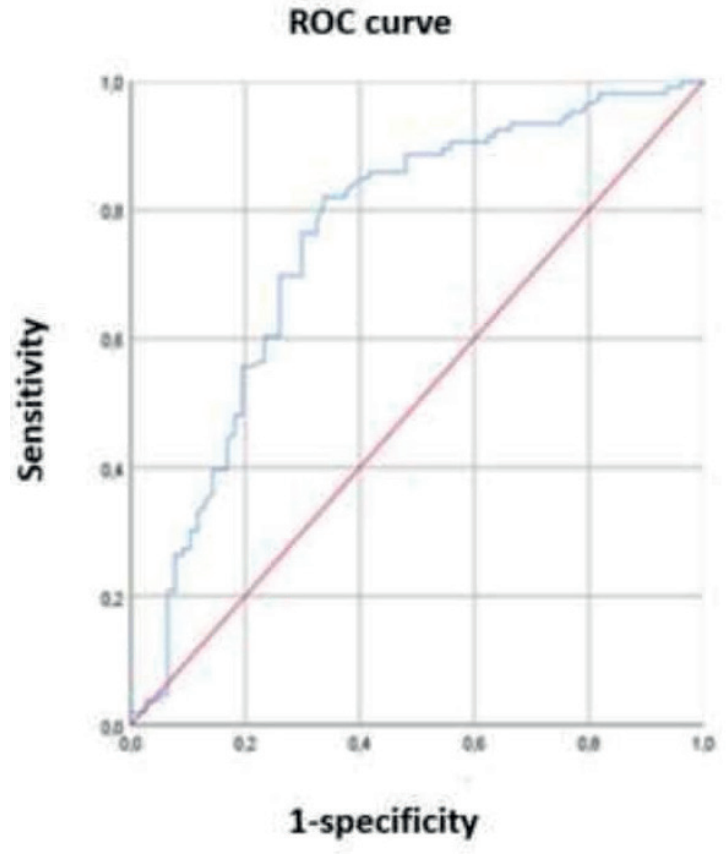

Fig. 3. Diagnostic value of fecal elastase in predicting FAP-NOS (AUC: $0.753,95 \%$ CI: 0.678-0.827, $p<0.001$ ).

we designed the present study based on the hypothesis that PEI could be a cause of FAPNOS.
Pancreatic exocrine functions are commonly assessed based on the levels of enzymes including lipase, pancreatic amylase, fecal elastase, and trypsinogen. In the present study, we measured the levels of these enzymes and found no significant difference between the study and control groups with regard to lipase, pancreatic amylase, and trypsinogen levels while serum amylase levels were significantly higher in the study group compared to the control group. Similarly, Feng et al. ${ }^{14}$ evaluated patients undergoing oral doubleballoon enteroscopy and reported that serum amylase levels were significantly increased in all the patients. The authors also noted that hyperamylasemia correlated well with increased intestinal permeability associated with bowel inflammation. Meaningfully, clinical conditions including increased concentrations of mucosal proinflammatory cytokines, gut microbiota alterations, increased intestinal permeability, and impaired intestinal motility have been blamed in the etiopathogenesis of FAP, all of which ultimately result in chronic abdominal inflammation. ${ }^{15}$ Accordingly, we consider that the increased amylase levels detected in our study group could not have a pancreatic origin and could be associated with bowel wall inflammation, a condition which is also included the pathophysiology of FGID. Moreover, this hypothesis can be supported by the normal levels of pancreatic amylase and lipase detected in both groups.

Fecal elastase-1 (FE-1) test could be a viable first-step test in the evaluation of pancreatic functions due to its reliability, practicality, and cost-effectiveness. In the present study, FE-1 test was performed to assess pancreatic insufficiency and it was revealed that the FE-1 levels were significantly lower in the study group compared to the control group. On the other hand, it is commonly known that patients with chronic pancreatic insufficiency and low FE-1 levels typically have dysbiosis as well. ${ }^{16}$ Additionally, Salvatore et al. ${ }^{17}$, detected low FE-1 levels in $28 \%$ of patients with infectious enteritis which demonstrated that low FE-1 levels could 
also be seen in conditions characterized by impaired gut microbiota such as infectious enteropathies. In a similar way, Stein et al. ${ }^{18}$ also detected low FE-1 levels in patients with malabsorption symptoms such as diarrhea and weight loss. Accumulating evidence suggests that PEI may also develop secondary to gastric surgery and other malabsorption symptoms such as celiac disease, Zollinger-Ellison syndrome, and human immunodeficiency virus (HIV) infection. ${ }^{19-21}$ In such patients, pancreatic enzyme replacement therapy (PERT) has been shown to reduce malabsorption by altering intestinal microbiota, inhibiting bacterial overgrowth in the duodenum and jejunum, and increasing the intestinal absorptive capacity. ${ }^{21}$ Pezzilli et al.22 evaluated patients with pancreatic diseases and found increased fecal calprotectin levels and decreased FE-1 levels in the patients. The authors concluded that the impaired intestinal microbiota in patients with pancreatic insufficiency could be associated with intestinal inflammation. On the other hand, dysbiosis is also blamed in the pathophysiology of FGID. ${ }^{23,24}$ Based on these findings, we consider that the decreased FE-1 levels detected in our study group might be associated with malabsorption and inflammation secondary to dysbiosis. Moreover, the absence of a significant correlation between FE-1 and pancreatic amylase, lipase, and IRT levels implicates that PEI could be a secondary condition rather than a primary condition.

In our study, the cut-off value detected in ROC analysis for the diagnostic value of FE-1 in predicting FAP-NOS was found to be 140.107 $\mu \mathrm{g} / \mathrm{g}$. According to this value, the sensitivity, specificity, PPV, and NPV of FE-1 were $82.1 \%$, $66.2 \%, 77 \%$, and $73 \%$, respectively. In the literature, there are numerous sensitivity and specificity values reported for FE-1. Löser et al. ${ }^{25}$ reported that the sensitivity of FE-1 in predicting PEI was $63 \%$ for mild PEI and 100\% for moderate and severe PEI and its specificity was $93 \%$ for all forms of PEI. Siegmund et al. ${ }^{26}$ reported that the sensitivity of FE-1 was 54\% for mild PEI, 75\% for moderate PEI, and 79\% for severe PEI. Wali et al. ${ }^{27}$ reported that the sensitivity and specificity of FE- 1 were $41.7 \%$ and $49.2 \%$, respectively. On the other hand, some other studies showed that the sensitivity and specificity of FE-1 at a cut-off value of 200 $\mu \mathrm{g} / \mathrm{gr}$ ranged between $63.77 \%-89.5 \%$ and $93 \%$ $99 \%$, respectively. ${ }^{28,29}$ Although all these values have been reported for PEI, to our knowledge, there have been no studies reporting on the cutoff, sensitivity, and specificity values of FE-1 in predicting FAP-NOS. In our study, for the first time in the literature, the cut-off, sensitivity, and specificity values of FE- 1 in predicting FAP-NOS were calculated and the analyses indicated that the likelihood of FE-1 in providing a positive value in patients with FAP-NOS was almost 9 times higher than in individuals without FAPNOS, which was a remarkably high value.

In conclusion, the underlying causes of FAP in children remain unclear although a number of factors including increased concentrations of mucosal proinflammatory cytokines, gut microbiota alterations, increased intestinal permeability, and impaired intestinal motility have been blamed in the etiopathogenesis of FAP. In the present study, FE-1 levels were significantly lower in the study group compared to the control group and we consider that this difference could be attributed to malabsorption secondary to dysbiosis as there is not enough data about this. Considering that there is paucity in similar studies evaluating FAP in children, we suggest that further studies are needed to substantiate our findings.

\section{REFERENCES}

1. Saps M, Nichols-Vinueza DX, Rosen JM, VelascoBenítez CA. Prevalence of functional gastrointestinal disorders in Colombian School children. J Pediatr 2014; 164: 542-545.e1.

2. Devanarayana N.M, Mettananda S, Liyanarachchi C, et al. Abdominal pain-predominant functional gastrointestinal diseases in children and adolescents: prevalence, symptomatology, and association with emotional stress. J Pediatr Gastroenterol Nutr 2011; 53: 659-665. 
3. Hyams JS, Di Lorenzo C, Saps M, Shulman RJ, Staiano A, van Tilburg M. Childhood functional gastrointestinal disorders: child/adolescent. Gastroenterology 2016; 150: 1456-1468.e2.

4. Korterink J, Devanarayana NM, Rajindrajith S, Vlieger A, Benninga MA. Childhood functional abdominal pain: mechanisms and management. Nat Rev Gastroenterol Hepatol 2015; 12: 159-171.

5. Olsen TS. The incidence and clinical relevance of chronic inflammation in the pancreas in autopsy material. Acta Pathol Microbiol ScandA 1978; 86A: 361-365.

6. Lacy BE, Mearin F, Chang L, et al. Bowel disorders. Gastroenterology 2016; 150: 1393-1407.e5.

7. Leeds JS, Hopper AD, Sidhu R, et al. Some patients with irritable bowel syndrome may have exocrine pancreatic insufficiency. Clin. Gastroenterol Hepatol 2010; 8: 433-438.

8. Talley NJ, Holtmann G, Nguyen QN, et al. Undiagnosed pancreatic exocrine insufficiency and chronic pancreatitis in functional GI disorder patients with diarrhea or abdominal pain. J Gastroenterol Hepatol 2017; 32: 1813-1817.

9. World Health Organization (WHO). WHO's Child Growth Standards (Weight for age). (Available at: https://www.who.int/childgrowth/standards). (Accessed: 1 April 2019)

10. Layer P, Yamamoto H, Kalthoff L, Clain JE, Bakken LJ, DiMagno EP. The different courses of earlyand lateonset idiopathic and alcoholic chronic pancreatitis.Gastroenterology 1994; 107: 1481-1487.

11. Lindkvist B. Diagnosis and treatment of pancreatic exocrine insufficiency. World J Gastroenterol 2013; 19: 7258-7266.

12. DiMagno EP, Go VL, Summerskill WH. Relations between pancreatic enzyme outputs and malabsorption in severe pancreatic insufficiency. N Engl J Med 1973; 288: 813-815.

13. Othman MO, Harb D, Barkin JA. Introduction and practical approach to exocrine pancreatic insufficiency for the practicing clinician. Int J Clin Pract 2018; 72: e13066.

14. Feng N, Dai J, Lu H, Li XB, Gao YJ, Ge ZZ. Hyperamylasemia is associated with increased intestinal permeability in patients undergoing diagnostic oral double-balloon enteroscopy. World J Gastroenterol 2014; 20: 539-545.

15. Holtmann G, Shah A, Morrison M. Pathophysiology of functional gastrointestinal disorders: aholistic overview. Dig Dis 2017; 35(Suppl 1): 5-13.
16. Frost F, Kacprowski T, Rühlemann M, et al. Impaired exocrine pancreatic function associates with changes in intestinal microbiota composition and diversity. Gastroenterology 2019; 156: 1010-1015.

17. Salvatore S, Finazzi S, Barassi A, et al. Low fecal elastase: potentially related to transient small bowel damage resulting from enteric pathogens. J Pediatr Gastroenterol Nutr 2003; 36: 392-396.

18. Stein J, Schoonbroodt D, Jung M, Lembcke B, Caspary WF. Measurement of fecal elastase 1 by immunoreactivity: a new indirect test of the pancreatic function. Gastroenterol Clin Biol 1996; 20: 424-429.

19. Leeds JS, Hopper AD, Hurlstone DP, et al. Is exocrine pancreatic insufficiency in adult coeliac disease a cause of persisting symptoms? Aliment Pharmacol Ther 2007; 25: 265-271.

20. Chaudhary A, Domínguez-Muñoz JE, Layer $P$, Lerch MM. Pancreatic exocrine insufficiency as a complication of gastrointestinal surgery and the impact of pancreatic enzyme replacement therapy. Dig Dis 2020; 38: 53-68.

21. Carroccio A, Guarino A, Zuin G, et al. Efficacy of oral pancreatic enzyme therapy for the treatment of fat malabsorption in HIV-infected patients. Aliment Pharmacol Ther 2001; 15: 1619-1625.

22. Pezzilli R, Barassi A, Morselli-Labate AM, et al. Fecal calprotectin and elastase 1 determinations in patients with pancreatic diseases: a possible link between pancreatic insufficiency and intestinal inflammation. J Gastroenterol 2007; 42: 754-760.

23. Rhee SH, Pothoulakis C, Mayer EA. Principles and clinical implications of the brain-gut-enteric microbiota axis. Nat Rev Gastroenterol Hepatol 2009; 6: 306-314

24. Ohman L, Simren M. Intestinal microbiota and its role in irritable bowel syndrome (IBS). Curr Gastroenterol Rep 2013; 15: 323.

25. Löser C, Möllgaard A, Fölsch UR. Faecal elastase 1: a novel, highly sensitive, and specific tubeless pancreatic function test. Gut 1996; 39: 580-586.

26. Siegmund E, Löhr JM, Schuff-Werner P. The diagnostic validity of non-invasive pancreatic function tests-a meta-analysis. Z Gastroenterol 2004; 42: 1117-1128.

27. Wali PD, Loveridge-Lenza B, He Z, Horvath K. Comparison of fecal elastase-1 and pancreatic function testing in children. J Pediatr Gastroenterol Nutr 2012; 54: 277-280. 
28. Dominguez-Munoz JE, Hieronymus C, Sauerbruch $T$, Malfertheiner P. Fecal elastase test: evaluation of a new noninvasive pancreatic function test. Am J Gastroenterol 1995; 90: 1834-1837.
29. Gullo L, Ventrucci M, Tomassetti P, Migliori M, Pezzilli R. Fecal elastase 1 determination in chronic pancreatitis. Dig Dis Sci 1999; 44: 210-213. 